\title{
Sum-frequency generation in sol-gel material doped with binaphthol
}

Grégory Taupier, May Saad, Michelangelo Romeo, Olivier Crégut, Alex Boeglin, Loic Mager, Alberto Barsella, Hicham Arhach, Jean-Luc Rehspringer, and Kokou D. (Honorat) Dorkenoo

Citation: Appl. Phys. Lett. 101, 241911 (2012); doi: 10.1063/1.4772001

View online: https://doi.org/10.1063/1.4772001

View Table of Contents: http://aip.scitation.org/toc/apl/101/24

Published by the American Institute of Physics

\section{Articles you may be interested in}

Optically active sum-frequency generation as an advanced tool for chiral metallopolymer material Applied Physics Letters 110, 021904 (2017); 10.1063/1.4973580

Vibrational spectroscopy of interfaces by infrared-visible sum frequency generation Journal of Vacuum Science \& Technology A: Vacuum, Surfaces, and Films 19, 2717 (2001); 10.1116/1.1414120

Compact, high-repetition-rate source for broadband sum-frequency generation spectroscopy APL Photonics 2, 066102 (2017); 10.1063/1.4983691

Enhanced ordering of water at hydrophobic surfaces

The Journal of Chemical Physics 140, 054711 (2014); 10.1063/1.4863558

Observation of buried water molecules in phospholipid membranes by surface sum-frequency generation spectroscopy

The Journal of Chemical Physics 131, 161107 (2009); 10.1063/1.3257600

\section{PHYSICS TODAY}

MANAGER'S GUIDE WHITEPAPERS

\section{READ NOW}

PRESENTED BY

Accelerate R\&D with
Multiphysics Simulation 


\title{
Sum-frequency generation in sol-gel material doped with binaphthol
}

\author{
Grégory Taupier, May Saad, Michelangelo Romeo, Olivier Crégut, Alex Boeglin, Loic Mager, \\ Alberto Barsella, Hicham Arhach, Jean-Luc Rehspringer, \\ and Kokou D. (Honorat) Dorkenoo a) \\ Institut de Physique et Chimie des Matériaux de Strasbourg, Université de Strasbourg, CNRS UMR 7504, \\ 23 rue du Loess, BP 43, F-67034 Strasbourg Cedex 2, France
}

(Received 24 July 2012; accepted 28 November 2012; published online 12 December 2012)

\begin{abstract}
A sum-frequency imaging system is used to investigate optical activity in a sol-gel material doped with the chiral molecule binaphthol. We show that this material can be optically structured to embed information that can be retrieved by sum-frequency. (C) 2012 American Institute of Physics.

[http://dx.doi.org/10.1063/1.4772001]
\end{abstract}

It is often stated that materials can only produce a coherent second-order nonlinear optical response when they are non-centrosymmetric on a macroscopic scale. Although most liquids, amorphous materials, sol-gels, and polymers are centrosymmetric, Shen and co-workers ${ }^{1,2}$ have recently demonstrated that second-order effects can be generated in liquid media when they exhibit optical activity. Meanwhile, polymers or sol-gels doped with optically active molecules have been intensively studied in the last decade in the search for superior materials for optical storage systems. In the race to achieve a high capacity storage device, polymethyl methacrylate (PMMA) based holographic materials of high optical quality and mechanical stability have been used to demonstrate the recording of images in large volumes. ${ }^{3}$ But in order to overcome the limitations in the fabrication rate of such holographic devices, many original recording schemes have been proposed. In this respect, recording techniques based on molecular photochromes have been proposed by different research groups. ${ }^{4,5}$ In this work, we have induced chirality in a sol-gel material by doping it with the chiral binaphthol (BN) molecule. We have used sum-frequency generation (SFG) microscopy to locally monitor the evolution of the trapped population of its $\mathrm{R}$ enantiomer. In the course of this study, we have devised a simple optical recording device based on irreversible changes in the material during irradiation.

The quadratic optical phenomenon of SFG originates in the first hyperpolarizability $\beta_{x y z}\left(-\omega_{s} ; \omega_{2}, \omega_{1}\right)$ of the chromophore, where $x, y$, and $z$ stand for molecular axes and where $\omega_{s}=\omega_{2}+\omega_{1}$. Notice that here we assume that we can work in the electric dipole approximation. For an enantiomeric population of chiral molecules in solution, one defines an orientationally averaged hyperpolarizability

$$
\langle\beta\rangle=\left[\beta_{x y z}-\beta_{y x z}+\beta_{y z x}-\beta_{z y x}+\beta_{z x y}-\beta_{x z y}\right] / 6,
$$

which would be vanishing by Kleinman symmetry if it was not for resonance effects: at least one of the three frequencies $\omega_{1}, \omega_{2}$, or $\omega_{s}$ has to fall within an absorption band of the chromophore. Assuming that this is only the case for $\omega_{s}$, the general microscopic expression for $\beta_{x y z}\left(-\omega_{s} ; \omega_{2}, \omega_{1}\right)$ leads to

${ }^{\text {a)} E l e c t r o n i c ~ a d d r e s s: ~ k d o r k e n o @ i p c m s . u n i s t r a . f r . ~}$

$$
\langle\beta\rangle=\frac{e^{3}}{6 \hbar^{2} \epsilon_{0}} \sum_{n, m} \frac{\omega_{1}-\omega_{2}}{\omega_{s}-\omega_{n g}+i \gamma_{n g}} \frac{\vec{\mu}_{g n} \cdot\left(\vec{\mu}_{n m} \times \vec{\mu}_{m g}\right)}{\left(\omega_{1}-\omega_{m g}\right)\left(\omega_{2}-\omega_{m g}\right)},
$$

where $g, n$, and $m$ denote the ground and two excited electronic states, respectively. ${ }^{1}$ Energy differences between states $i$ and $j$ are written $\omega_{i j}$, while $\gamma_{i j}$ and $\vec{\mu}_{i j}$ stand for the corresponding homogeneous width and transition dipole moment. Notice that $\langle\beta\rangle$ changes sign upon mirror symmetry because of the scalar triple product between the $\vec{\mu}$ vectors which reflects the chiral nature of the response.

When two transverse electric input fields $\vec{E}_{1}(\vec{r})$ and $\vec{E}_{2}(\vec{r})$ propagate through the chiral medium as plane waves of frequencies $\omega_{1}$ and $\omega_{2}$, the source of the SFG signal is the induced polarization

$$
\vec{P}^{S F G}(\vec{r})=\chi^{(2)}\left(-\omega_{s} ; \omega_{2}, \omega_{1}\right)\left[\vec{E}_{1}(\vec{r}) \times \vec{E}_{2}(\vec{r})\right],
$$

where $\chi^{(2)}$ is the only independent component of the quadratic optical susceptibility tensor. Thus, if both optical fields were to propagate collinearly, they could only give rise to a longitudinal polarization which cannot be the source of a propagating sum-frequency field. However, if the incoming fields propagate along two distinct directions and are polarized in their common plane of incidence (PP), they will produce a transverse polarization (S) in the volume where they intersect. $^{7}$

Thus, from an experimental point of view, one can work in the so called wave-mixing geometry and we have been able to use our two-photon microscopy setup as described in our previous work. ${ }^{6}$ It is similar to the one described by Shen and co-workers ${ }^{2}$ and is schematically shown in Fig. 1. Laser pulses of $80 \mathrm{fs}$ duration are delivered at $80 \mathrm{MHz}$ repetition rate by a Ti:sapphire at $900 \mathrm{~nm}$. The laser beam is separated into two parts, one generating the pump beam at $450 \mathrm{~nm}$ by second harmonic generation in a $1 \mathrm{~mm}$ thick beta barium borate (BBO) crystal. These two beams are collimated and directed into a modified commercial microscope (Olympus IX71) with a center-to-center parallel displacement of $5 \mathrm{~mm}$. They are focused on the sample through a microscope objective (Olympus PlanApo, 60×, 1.42 NA, oil immersion). The SFG output from the sample is collected in the forward direction by a $\mathrm{CaF}_{2} \mathrm{UV}$ objective (LMU $40 \times$ UVB). For spectral analysis of the sum-frequency output, a 


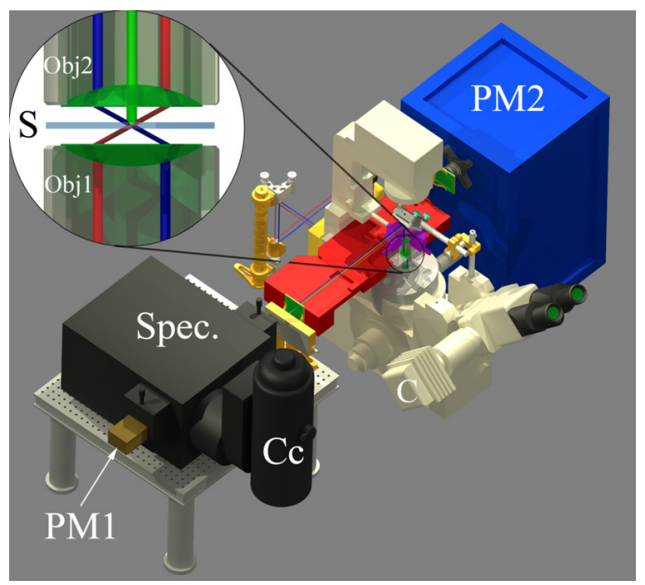

FIG. 1. Setup arrangement for SFG, SHG, TPA detection: $\mathrm{PM}_{1}$ and $\mathrm{PM}_{2}$ are photomultipliers, $\mathrm{Obj}_{1}$ and $\mathrm{Obj}_{2}$ microscope objectives, $\mathrm{S}$ is the sample position, CC the cooled CCD camera, $\mathrm{C}$ is the alignment camera, Spec. is the spectrometer.

spectrograph (Acton Research, SP2300) is used. The two dimensional imaging scans are performed by a motorized XYZ sample stage (Marzhauser Scan IM) combined with a photomultiplier (H7467-03).

The sol-gel was prepared using tetraethoxysilane (TEOS) in solution in n-butanol with a molar concentration of $3 \mathrm{moll}^{-1}$. N-butanol was chosen due to its good ability to dissolve binaphthol. TEOS was hydrolysed using a $0.01 \mathrm{M}$ $\mathrm{HNO}_{3}$ water solution. The ratio of TEOS to water was about 4. $60 \mathrm{mg}$ of binaphthol was dissolved in $1 \mathrm{ml}$ of sol-gel preparation. $5 \mu \mathrm{l}$ of this solution was deposited on a glass substrate, covered with a glass plate, and left to dry slowly. Solvents progressively diffuse towards the edges of the glass plate to vaporize and gelation occurs. A glassy composite is obtained. A strong Si-O-Si network appears due to condensation between hydroxy silane produced during the hydrolysis process (water addition). A micro porous network appears with the first silica condensations and evolves gradually as the condensation reactions progress. These further reactions lead also to a shrinkage of the network leading chiefly to a thinning of the film without affecting its optical quality.

In our experiments, we have used two combinations of output/input polarizations. The two incident beams $\omega_{2}$ and $\omega_{1}$ $(450 \mathrm{~nm}$ and $900 \mathrm{~nm})$ are P-polarized, while a motorized polarizer selects either the S- or the P-polarization of the output $\omega_{S}$ $(300 \mathrm{~nm})$ thus switching between the SPP and PPP modes. In order to test our setup, we have chosen to work first with a sample similar to the one described by Ji et al. ${ }^{2}$ Thus, a drop of tetrahydrofuran (THF) solution of BN (130 g/l) was sandwiched between two fused silica plates. The UV absorption spectrum of this solution is shown in Fig. 2(a). The signal detected by the CCD is shown in Fig. 2(b), where the blue curve corresponds to the PPP configuration, while the red one corresponds to the SPP configuration. The sum frequency signal is observed at $300 \mathrm{~nm}$, within the $\mathrm{BN}$ absorption band, and is only detected in the SPP configuration. The linear dependence of the signal on the intensity of each of the incoming beams has been verified as Fig. 2(c) shows for the $450 \mathrm{~nm}$ beam. This result proves that no other effect such as four-wave mixing (FWM) or coherent anti-stokes Raman scattering $(\mathrm{CARS})^{8}$ contribute to the signal.

Our setup being thus validated, we proceeded to work on sol-gel samples prepared as describe above, BN doped at $60 \mathrm{~g} /$ 1 , and dried for a week. Working with $30 \mathrm{~mW}$ at $900 \mathrm{~nm}$ and with $1.3 \mathrm{~mW}$ at $450 \mathrm{~nm}$, the spectrum of the signal is identical to one of the liquid samples, but its intensity decreases during illumination. In order to quantify this decrease, we have irradiated successive $50 \times 50 \mu \mathrm{m}$ zones (using the motorized XYZ sample stage) for exposure times from 0 to $50 \mathrm{~ms}$. These zones have subsequently been scanned again for SFG signal during $20 \mathrm{~ms}$ for each pixel. Fig. 2(d) represents the total SFG a)
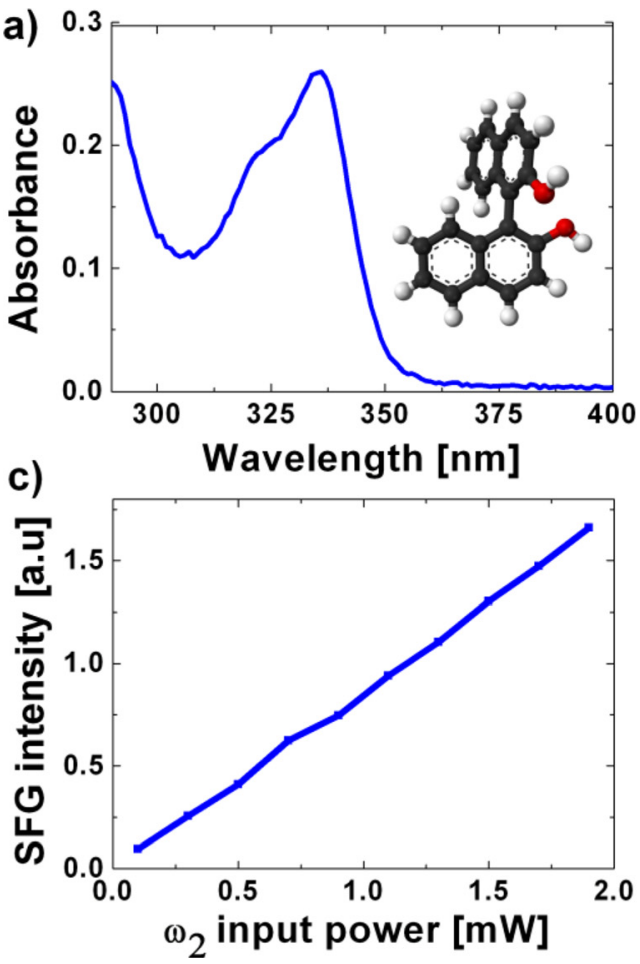
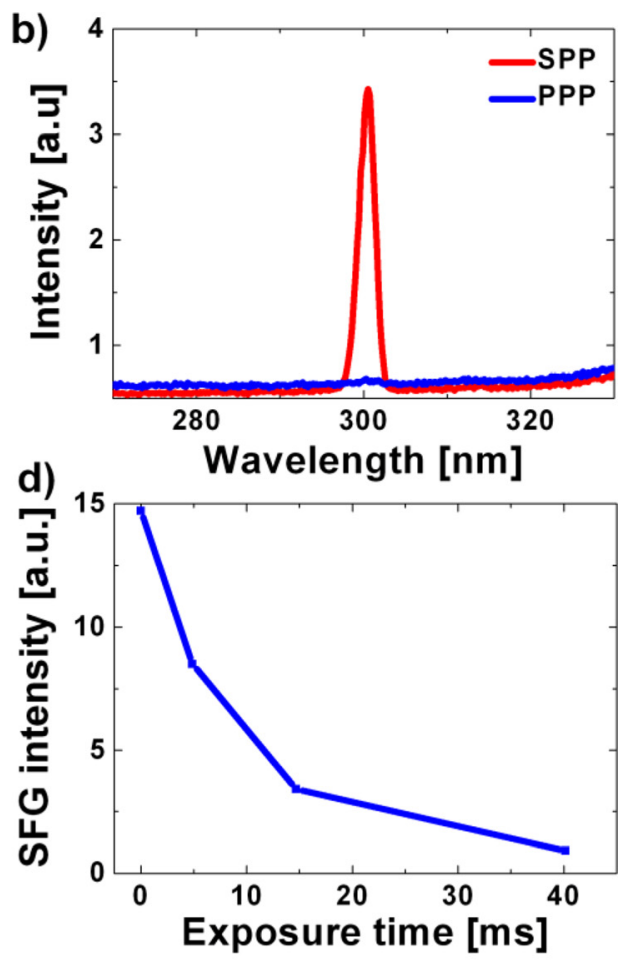

FIG. 2. (a) UV absorption spectrum of $0.45 \mathrm{moll}^{-1}$ of BN in THF, (b) SFG signal is observed only for the SPP polarization configuration, (c) SFG intensity versus input power of the beam at $450 \mathrm{~nm}$, (d) SHG intensity loss as a function of exposure time. 


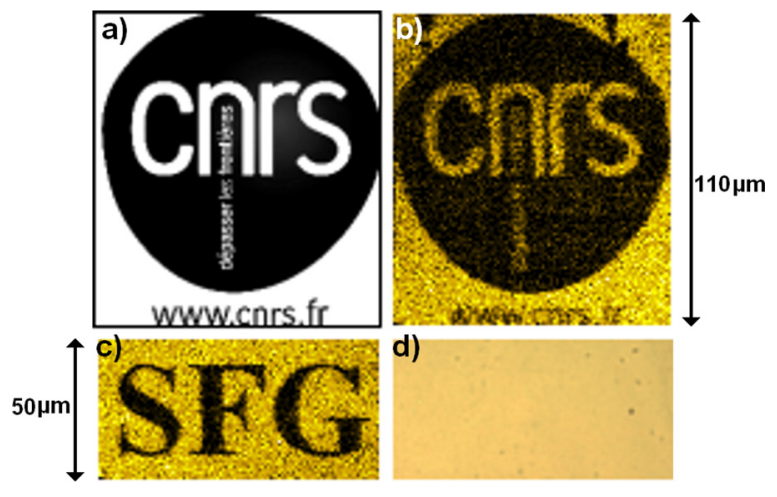

FIG. 3. (a) Pattern, (b) and (c) SFG chiral images, (d) optical microscope image.

intensity recorded in this way: less than $50 \mathrm{~ms}$ of exposure time is needed to erase the optical activity. In addition to the SFG process, the chromophores may also be promoted to an excited state through two-photon absorption processes. These excitation processes will eventually lead to the bleaching of chromophores and are, therefore, responsible for the drop in SFG signal during irradiation. Notice that these phenomena are not detected when working with liquid samples as diffusion processes continuously renew the chromophores in the excitation volume.

However, the above procedure makes it possible to calibrate operating intensities and sample characteristics in order to demonstrate the possibility of image inscription and retrieval as shown in Fig. 3. Starting from a pattern as shown in Fig. 3(a) and bleaching each pixel belonging to a black area, we can subsequently read out the picture again through SFG as shown in Fig. 3(b). Fig. 3(c) shows another such readout, while Fig. 3(d) corresponds to the usual microscopy picture of the same area taken in white light and showing no trace of induced changes in the sample.

Since photobleaching of the chiral chomophores is at the origin of the loss in the SFG intensity, we may exploit this process in order to achieve controlled modulation of signal levels. To this end, we may code gray levels by varying the exposure time. In Fig. 4, we show the results of this procedure when encoding the image of an airy disk into the doped sol-gel sample.

The resulting images obtained in the SPP and PPP polarization configurations are shown, respectively, in Figs. 4(a) and 4(b). Only the SPP configuration displays the inscribed
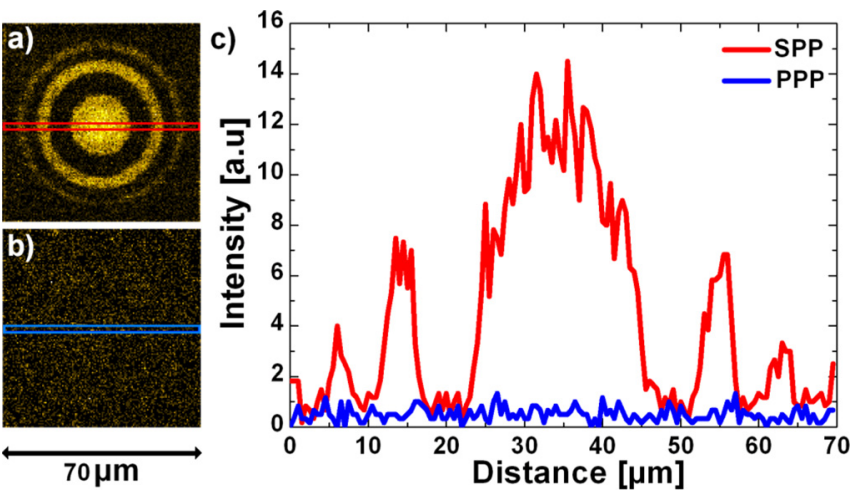

FIG. 4. SFG images of an airy disk (a) in SPP configuration, (b) in PPP configuration, (c) profiles of signal levels along the zone delimited in (a) and (b).

pattern. Fig. 4(c) shows the intensity profile along the zone delimited by two straight lines in Figs. 4(a) and 4(b).

In conclusion, we have extended the proposal of Shen to perform SFG imaging in chiral media from liquid samples containing neutral beads to solid samples embedded with chiral chromophores. In this way, we have shown that SFG experiments in the visible range reveal transient phenomena in BN molecules when they are immobilized in a transparent network. Some form of irreversible bleaching is induced by the two-photon absorption processes allowed by the pump beams. Because of this, we have been able to demonstrate the possibility of SFG imaging in a sol-gel environment.

K. Dorkenoo needs to thank Emefa Zinmonse for helpful discussions. This work was supported by ANR-BlancSIMI-4-2012 "DynaChir."

${ }^{1}$ M. A. Belkin, S. H. Han, X. Wei, and Y. R. Shen, Phys. Rev. Lett. 87, 113001 (2001).

${ }^{2}$ N. Ji, K. Zhang, H. Yang, and Y.-R. Shen, J. Am. Chem. Soc. 128, 34823483 (2006).

${ }^{3}$ D. Gindre, I. Ka, A. Boeglin, A. Fort, and K. D. Dorkenoo, Appl. Phys. Lett. 90, 094103 (2007).

${ }^{4}$ F. M. Raymo and S. Giordani, Proc. Natl. Acad. Sci. U.S.A. 99, 4941-4944 (2002).

${ }^{5}$ M. Irie, Chem. Rev. 100, 1683-1890 (2000).

${ }^{6}$ C. Doras, G. Taupier, A. Barsella, L. Mager, A. Boeglin, H. Bulou, P. Bousquet, and K. D. Dorkenoo, Opt. Express 19, 15062 (2011).

${ }^{7}$ P. Fischer, K. Beckwitt, F. W. Wise, and A. C. Albrecht, Chem. Phys. Lett. 352, 463 (2002).

${ }^{8}$ P. Fischer, D. S. Wiersma, R. Righini, B. Champagne, and A. D. Buckingham, Phys. Rev. Lett. 85(20), 4253 (2000). 